\title{
Application of the Breakthrough RESEARCH social and behavior change costing guidelines to the RISE II Project in Niger
}

Nicole M. Bellows

Lori Bollinger

Rudolph Chandler

Follow this and additional works at: https://knowledgecommons.popcouncil.org/departments_sbsr-pgy

Part of the Health Communication Commons, and the Public Health Commons

How does access to this work benefit you? Let us know!

\section{Recommended Citation}

Bellows, Nicole M., Lori Bollinger, and Rudolph Chandler. 2021. "Application of the Breakthrough RESEARCH social and behavior change costing guidelines to the RISE II Project in Niger," Breakthrough RESEARCH Technical Report. Washington, DC: Population Council. 


\section{TECHNICAL REPORT}

\section{Application of the}

Breakthrough RESEARCH

Social and Behavior Change

Costing Guidelines to the

RISE-II Project in Niger

FEBRUARY 2021

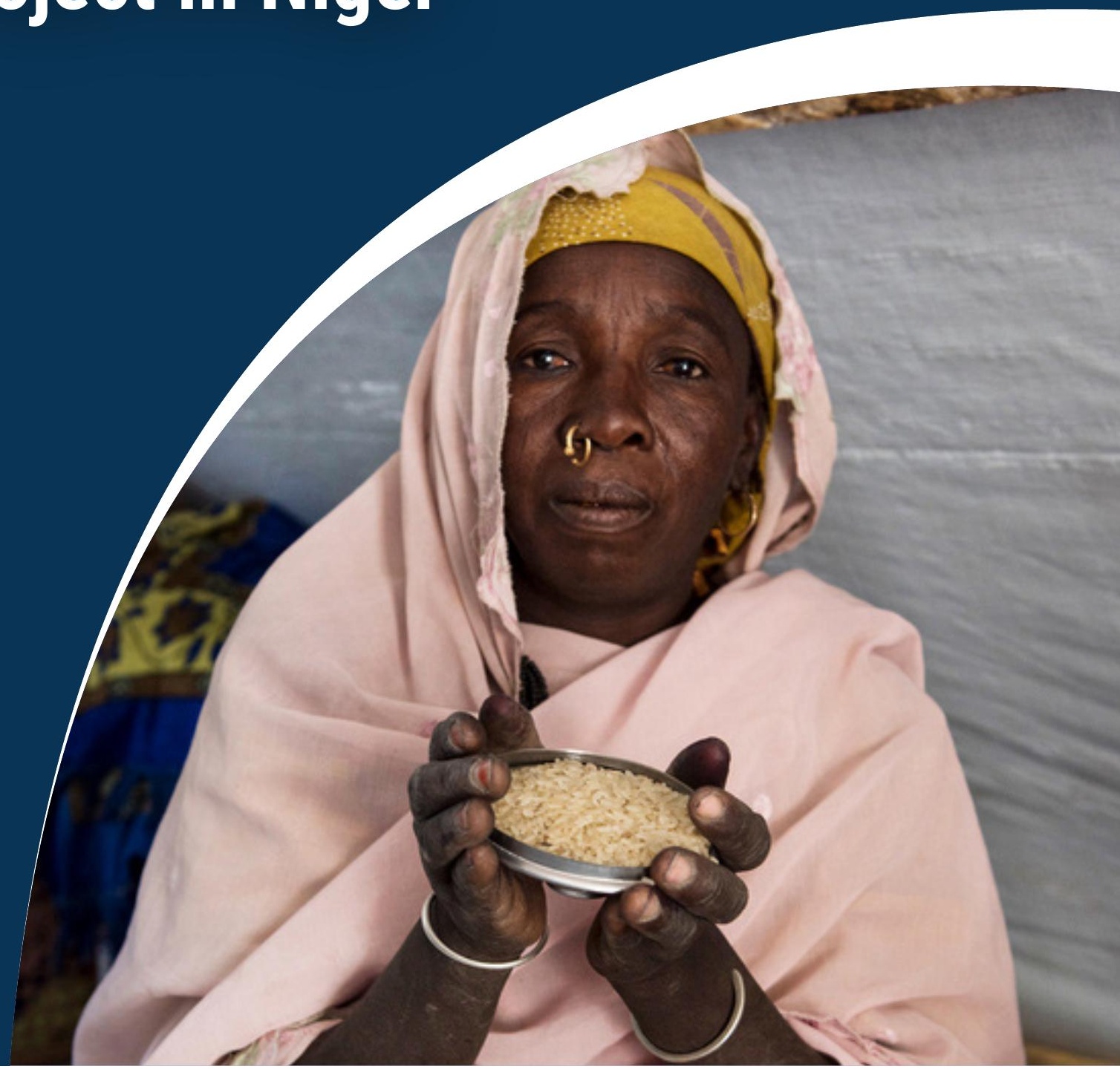




\section{Acknowledgments}

We would like to say thank you to the Resilience Food Security Activities in Niger which include Hamzari (led by Care), Girma (led by Catholic Relief Services), and Wadata (led by Save the Children) for their collaboration in this activity.

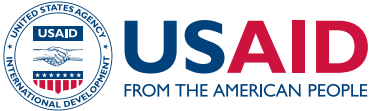

Breakthrough RESEARCH is made possible by the generous support of the American people through the United States Agency for International Development (USAID) under the terms of cooperative agreement no. AID-OAA-A-17-00018. The contents of this document are the sole responsibility of the Breakthrough RESEARCH and Population Council and do not necessarily reflect the views of USAID or the United States Government.

\section{- POPULATION COUNCIL}

Ideas. Evidence. Impact.

The Population Council confronts critical health and development issuesfrom stopping the spread of HIV to improving reproductive health and ensuring that young people lead full and productive lives. Through biomedical, social science and public health research in about 50 countries, the Council works with our partners to deliver solutions that lead to more effective policies, programs, and technologies to improve lives worldwide. Established in 1952 and headquartered in New York, the Council is a nongovernmental, nonprofit organization with an international board of trustees.

\section{Breakthrough
RESEARCH
FOR SOCIIL \& BEAAVIOR CHANGE}

Breakthrough RESEARCH catalyzes social and behavior change (SBC) by conducting state-of-the-art research and evaluation and promoting evidence-based solutions to improve health and development programs around the world. Breakthrough RESEARCH is a consortium led by the Population Council in partnership with Avenir Health, ideas42, Institute for Reproductive Health at Georgetown University, Population Reference Bureau, and Tulane University.

\section{Avenir Health}

Avenir Health was founded in 2006 as a global health organization that works to enhance social and economic development by providing tools and technical assistance in policy, planning, resource allocation and evaluation. Its focus is on developing and implementing demographic, epidemiological and costing models for long-range planning to assist with setting goals, strategies, and objectives. Avenir Health assists in both developing and implementing programs in HIV/AIDS, reproductive health, maternal health and other programming areas. Avenir Health works with government agencies, foundations, corporations, and nongovernmental organizations around the world.
(C)2021 The Population Council. All rights reserved.

Cover photo by USAID (CC BY-NC 2.0)

\section{Suggested Citation}

Bellows, Nicole, Lori Bollinger, and Rudolph Chandler. 2021. "Application of the Breakthrough RESEARCH Social and Behavior Change Costing Guidelines to the RISE-II Project in Niger," Breakthrough RESEARCH Technical Report. Washington, DC: Population Council.

\section{Contact}

4301 Connecticut Avenue NW, Suite 280 | Washington, DC 20008 +12022379400 | BreakthroughResearch@popcouncil.org breakthroughactionandresearch.org 


\section{Application of the Breakthrough RESEARCH Social and Behavior Change Costing Guidelines to the RISE-II Project in Niger}

Nicole Bellows 


\section{Acronyms}

ANC

Antenatal care

CEA

Cost-effective analysis

DALY Disability-adjusted life year

FP

Family planning

ICER

Incremental cost-effectiveness ratio

LiST

Lives Saved Tool

$\mathrm{MNCH}$

Maternal, newborn, and child health

RFSA

Resilience food security assistance

RISE

Resilience in the Sahel

SBC

Social and behavior change

UCSR

Unit Cost Study Repository

USAID

United States Agency for International Development

WASH

Water, sanitation, and hygiene 


\section{Table of Contents}

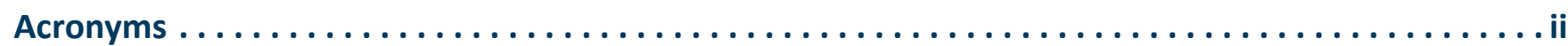

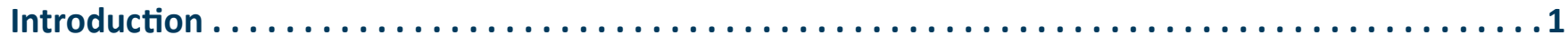

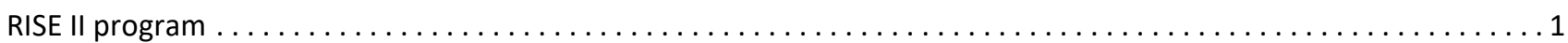

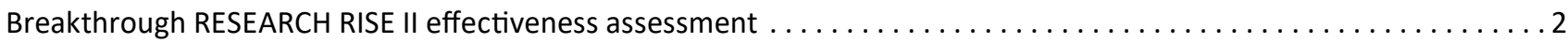

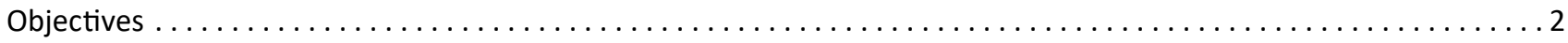

Breakthrough RESEARCH SBC Costing Guidelines $\ldots \ldots \ldots \ldots \ldots \ldots \ldots \ldots \ldots \ldots \ldots \ldots$

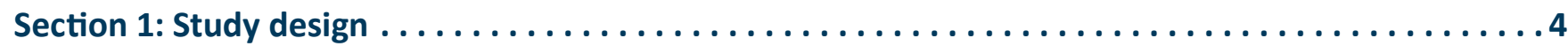

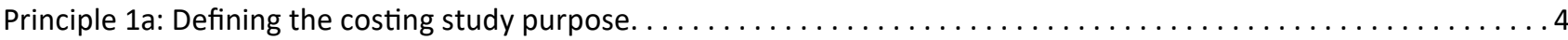

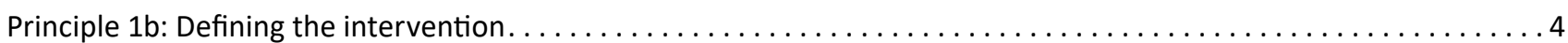

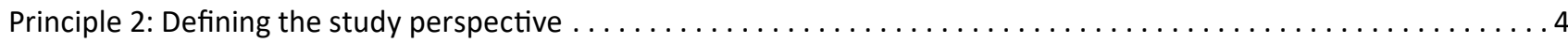

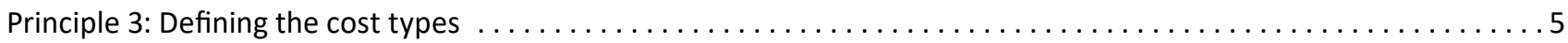

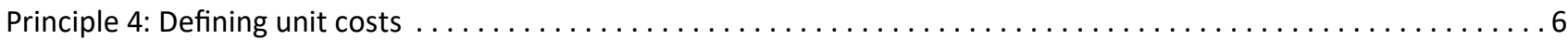

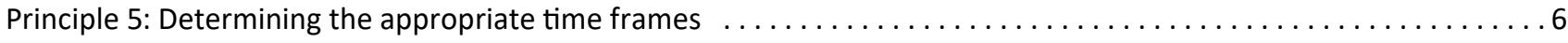

Section 2: Resource use measurement. . . . . . . . . . . . . . . . . . . .

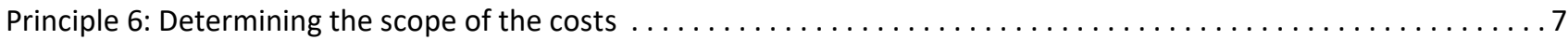

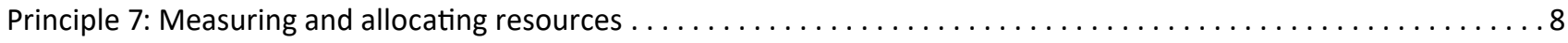

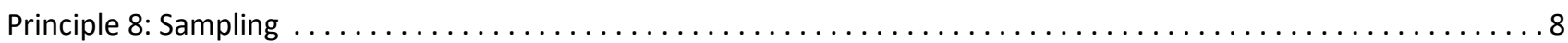

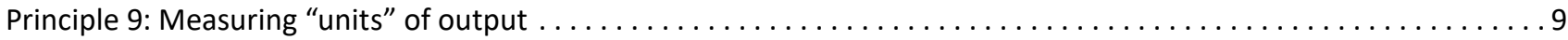

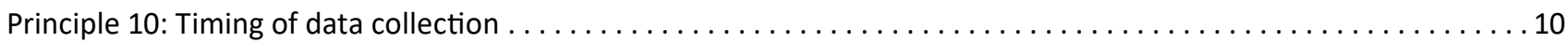

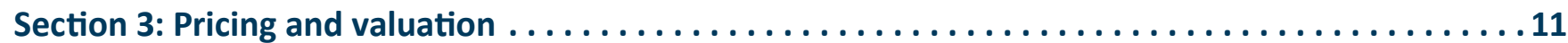

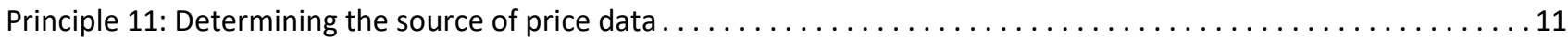

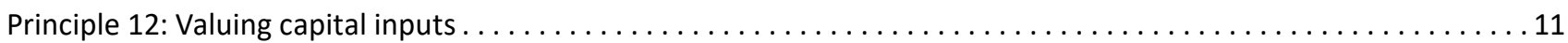

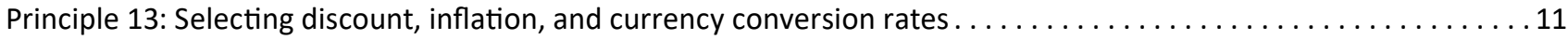

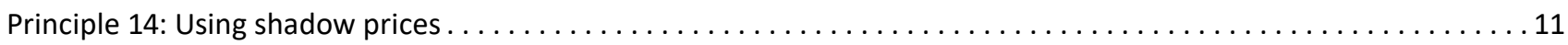

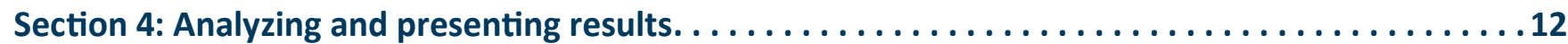

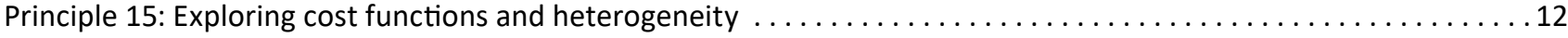

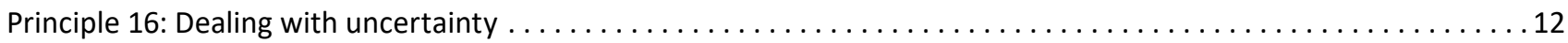

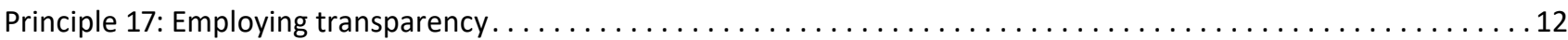

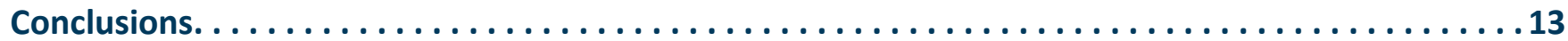

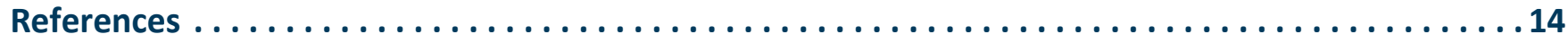




\section{Introduction}

Understanding the costs of social and behavior change (SBC) interventions is important for budgeting, price setting, and conducting cost-effectiveness studies, which can inform policy decisions to efficiently achieve health and development goals. The United States Agency for International Development (USAID)-funded Breakthrough RESEARCH project is providing evidence on the costs and impacts of SBC interventions and strengthening the case that investing in $\mathrm{SBC}$ is crucial for improving health and advancing development. As part of this work, Breakthrough RESEARCH drafted the Guidelines for Costing Social and Behavior Change Health Interventions, in consultation with its sibling project Breakthrough ACTION and other SBC implementers with the aim to promote high-quality SBC costing studies for a range of uses including budgeting, planning, economic evaluation, and advocacy. ${ }^{1}$

\section{BOX 1: DEFINITION OF KEY TERMS}

\section{Social behavior change interventions (SBC)-} interventions that seek to improve health-seeking behaviors of individuals and communities, as well as the norms that underpin those behaviors. ${ }^{2}$

Cost-effectiveness analysis (CEA)-examines the costs and health outcomes associated with one or more interventions to compare results against the status quo or between interventions. ${ }^{3}$

Integrated SBC-SBC interventions designed to cohesively address more than one health or development issue within the same program. ${ }^{4}$

In March 2020, Breakthrough RESEARCH began applying these guidelines to the Resilience in the Sahel (RISE) II program in Niger for a cost-effectiveness analysis (CEA) that will run until 2022. The purpose of this document is to share the findings of the application of the guidelines and provide an example and guidance for SBC researchers and implementers on how these guidelines can be applied to other case study applications.

\section{RISE II program}

RISE focuses on building the capacities of individuals, communities, and systems among chronically vulnerable populations in Niger and Burkina Faso, to help develop their resilience to environmental shocks and achieving eventual self-reliance. Initially conceived in 2012, the RISE program was developed by USAID as a response to a historical pattern of severe droughts and recurring environmental shocks and stressors that undermined development progress. ${ }^{5}$ For its first five years, RISE focused its efforts on strengthening state institutions and local governance, increasing sustainable economic well-being, and improving health and nutrition among its populations. ${ }^{5}$ These activities were integrated within existing humanitarian and development assistance to

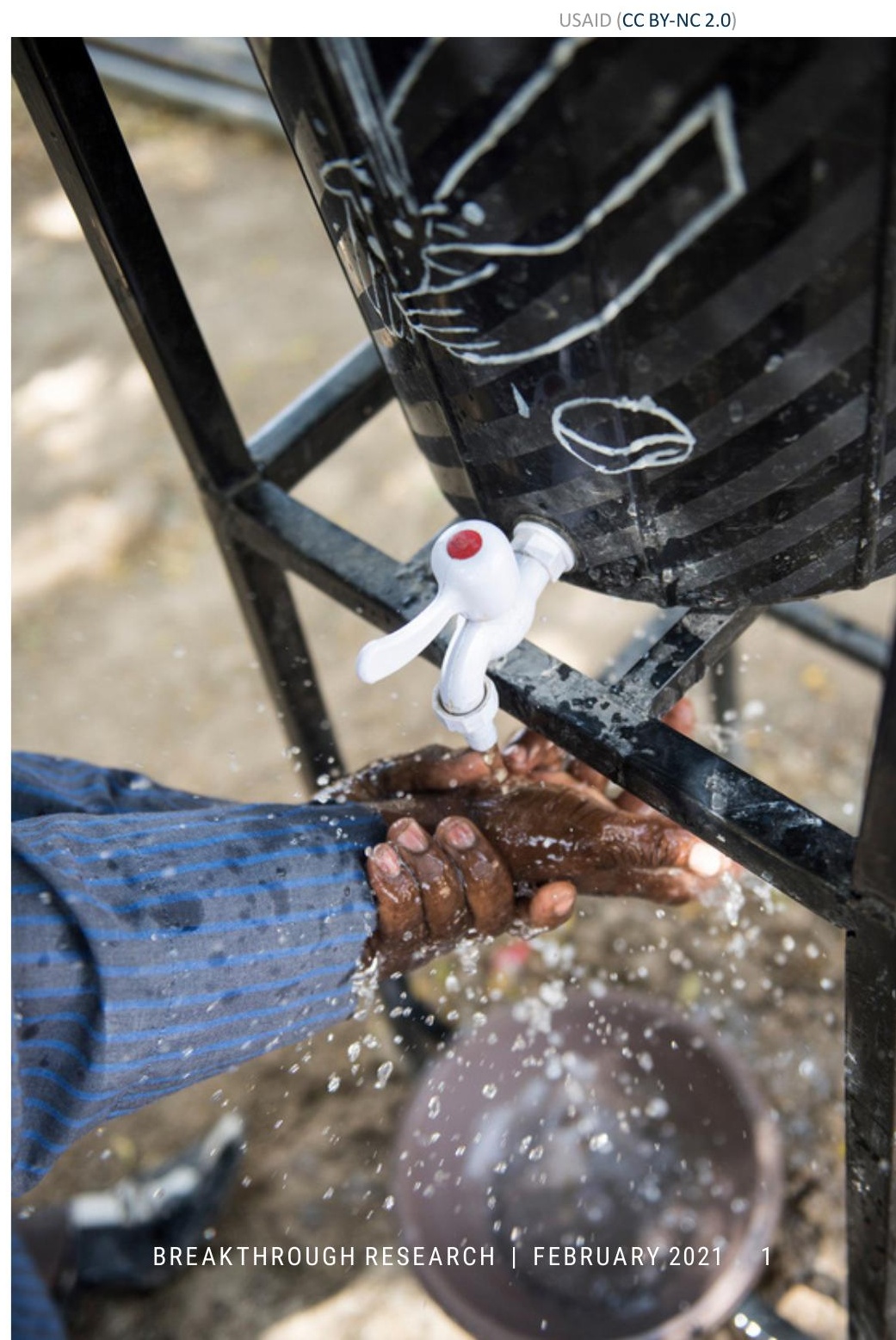


support those most vulnerable along with reducing the need for future humanitarian assistance.

USAID's RISE II project builds upon RISE's initial investments by expanding its SBC program through an integrated SBC strategy for improving priority behaviors and resultant health outcomes in maternal, newborn and child health (MNCH), family planning (FP), nutrition, and water, sanitation and hygiene (WASH). ${ }^{5}$ RISE II not only combines integrated service delivery and SBC programming in several health areas but in various sectors of society-civil and government-as well, to support effective social empowerment, productive livelihoods, and sustainable agricultural programming.

RISE II SBC interventions, developed in a participatory process with relevant stakeholders, from October 2019 to September 2020, are beginning implementation by Resilience Food Security Assistance (RFSA) partners through the establishment of groups for SBC activities in the near future. In Niger, the RFSAs are working in the Maradi and Zinder region and include Hamzari (led by Care), Girma (led by Catholic Relief Services), and Wadata (led by Save the Children). Breakthrough ACTION supports the development and implementation of SBC in RISE II through capacity building and leveraging their expertise on behavioral economics, human-centered design, and SBC implementation. There is a separate USAID-funded mechanism (the Kulawa activity) responsible for the health service delivery activities in the RISE II intervention zones, led by Save the Children.

RFSAs will focus on supporting community structures and groups, to facilitate comprehensive knowledge of people's barriers to improved health and social change with analysis to determine what factors are within a community's ability to influence. RFSAs will work with communities to identify and implement SBC approaches such as community mobilization, including peer group activities or community meetings, and improved interpersonal communication (IPC), with counseling paired with printed materials. RFSAs will support communities with coaching and mentoring approaches, such as community conversations, and will expand their work with women's care groups to other influential groups, such as male engagement and couples' work, savings and loan groups, youth theatre, and grandparent clubs. Due to the scope of the RISE II program, these approaches will be phased, with variations expected as SBC interventions are introduced in all three locations.

\section{Breakthrough RESEARCH RISE II effectiveness assessment}

As part of a broader evaluation, Breakthrough RESEARCH is conducting a repeated cross-sectional survey in the Maradi and Zinder regions that quantitatively assesses changes in ideational factors, attitudes, health intentions, decision making, and behaviors of women and men ages 15 to $49 .{ }^{6}$ The planned CEA will leverage the resulting data on health behaviors. Baseline behavioral health data will be captured prior to the delivery of the healthrelated SBC interventions and at endline. If SBC activities and the baseline survey continue to be delayed due to COVID-19, Avenir Health will work with project management to adapt a revised timeline for data collection and analysis.

\section{Objectives}

The objectives of this costing application exercise are twofold:

- Serve as a foundation for a costing and CEA study protocol that will examine the cost-effectiveness of integrated health SBC programming within RISE II in Niger.

- Guide implementers and researchers on applying Breakthrough RESEARCH SBC costing guidelines to their own programs and studies.

- Contribute to the greater SBC field by entering the unit cost findings into the Unit Cost Study Repository (UCSR), an online database of costing study results for interventions for HIV, tuberculosis, and SBC. 


\section{Breakthrough RESEARCH SBC Costing Guidelines}

Based on the Global Health Cost Consortium's Reference Case, and aligned with the USAID-funded Passages

Project's Social Norms Costing Primer, Breakthrough RESEARCH's Guidelines for Costing Social and Behavior Change Health Interventions were developed to provide needed guidance for SBC costing and enable high quality $\mathrm{SBC}$ costing studies to improve available evidence of SBC costs. ${ }^{7-9}$ Breakthrough RESEARCH's SBC costing guidelines include 17 methodological principles, in four sections:

- Study design

- Measuring use of resources

- Pricing and valuation

- Presentation of results

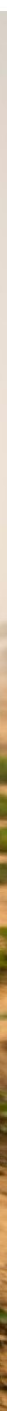




\section{SECTION 1 \\ Study Design}

A costing study begins with considering fundamental design questions in relation to the purposes and scope of the study. The five study design principles summarized below and discussed in this section are fundamental and underlie other data collection and presentation principles described in later sections of the guidelines.

\section{1a-Defining the study purpose}

$1 \mathrm{~b}-$ Defining the intervention

2-Defining the study perspective

3-Defining the type of costs(s)

4-Defining unit cost(s)

5-Determining the appropriate time frames

\section{Principle 1a: Defining the costing study purpose}

The first principle notes that an elemental step for any costing exercise is determining its specific purpose. Discussions between Breakthrough RESEARCH, Breakthrough ACTION, USAID, and RFSAs determined that the RISE II costing study has two primary purposes. First, it directly informs a CEA study of RISE II SBC health programming to determine its cost-effectiveness according to international standards. A second purpose is generating cost information for specific SBC activities that can improve program planning for RISE II, including mid-course decisions. The costing study will also contribute to the greater SBC field by entering the unit cost findings into the UCSR, an online database of costing study results for interventions for HIV, tuberculosis, and SBC. ${ }^{10}$ By including the results in the UCSR, the RISE II cost findings can subsequently be used by the international SBC community for future planning, budgeting, and advocacy.

\section{Principle 1b: Defining the intervention}

The second part of understanding the study purpose is ensuring awareness of the context in which the SBC intervention will take place and clearly understanding the intervention to be costed. Table 1 summarizes the various contextual factors and intervention characteristics to consider, as delineated in the guidelines and applied to RISE II.

One distinguishing feature of RISE II not captured by the guidelines is the multisectoral aspect of the program. The RISE II CEA will focus on health outcomes and SBC interventions most closely associated with achieving those health outcomes, but RISE II is also producing interventions for agricultural, livelihood, and other development outcomes. For the RISE II CEA, determining what is a health-related SBC intervention will be important for proper cost allocation. This will require discussions with RFSAs, Breakthrough ACTION, and USAID on the set of activities included and excluded from the costing, along with in-depth interviews paired with expenditure analysis (See Principle 7 for more details).

\section{Principle 2: Defining the study perspective}

This principle is critical in deciding which costs to include in the analysis, determined by the costing study's purpose. From an economics perspective, costing studies ideally employ a societal perspective encompassing a full accounting of all costs and effects, but most SBC costing studies adopt a provider perspective, focusing on the costs borne by implementing partners.

RISE II costing efforts will apply a provider perspective to determine the funding necessary to replicate the RISE II program in similar contexts and will include both in-country costs and any external costs for designing or implementing SBC activities. Costs associated with RFSAs, 


\section{TABLE 1 RISE II INTERVENTION DESCRIPTION}

\begin{tabular}{|c|c|}
\hline INTERVENTION CHARACTERISTICS & RISE II \\
\hline Country and geography & $\begin{array}{l}\text { The project is delivering integrated SBC in Niger and Burkina Faso. The focus of the cost- } \\
\text { ing study will be in the Maradi and Zinder regions of Niger. }\end{array}$ \\
\hline Epidemiological context & $\begin{array}{l}\text { The health indicators in the study area indicate high morbidity and mortality of adults } \\
\text { and children: high under-five child mortality ( } 84 \text { per } 1,000 \text { live births), high maternal mor- } \\
\text { tality (509 per 100,000 live births), and low life expectancy ( } 59.8 \text { years). Additionally, the } \\
\text { Niger fertility rate is the highest in the world at } 7 \text { children per woman. }{ }^{11} \text { Health behaviors } \\
\text { specific to the RISE II intervention are detailed in Principle } 9 .\end{array}$ \\
\hline Other contextual issues & $\begin{array}{l}\text { The Sahel experiences complex challenges, including extreme poverty, food insecurity, } \\
\text { climate shocks, violent extremism, and weak government structures. }\end{array}$ \\
\hline Target populations & $\begin{array}{l}\text { The program targets chronically vulnerable populations. The health behaviors targeted } \\
\text { in the cost-effectiveness study are primarily focused on the needs of vulnerable women } \\
\text { and children (e.g., antenatal care, breastfeeding, vaccinations). Men are targeted as family } \\
\text { leaders influencing health decision-making. }\end{array}$ \\
\hline Ecological level & $\begin{array}{l}\text { The ecological level for RISE II is at individual and community levels-trying to improve } \\
\text { health behaviors among individuals for themselves and their children, as well as building } \\
\text { capacity for self-reliance within a community. }\end{array}$ \\
\hline Delivery platform & $\begin{array}{l}\text { RISE II features multiple delivery platforms. A majority of the SBC intervention compo- } \\
\text { nents will be delivered at the community level through group meetings and mass media. }\end{array}$ \\
\hline Ownership & $\begin{array}{l}\text { SBC interventions are implemented by RFSAs: Hamzari (led by Care), Girma (led by Cath- } \\
\text { olic Relief Services), and Wadata (led by Save the Children). }\end{array}$ \\
\hline Main SBC intervention activities & $\begin{array}{l}\text { Primary interventions include IPC through peer activities, care group models, community } \\
\text { meetings, printed educational materials, and mass media. }\end{array}$ \\
\hline $\begin{array}{l}\text { Coverage level of population receiving } \\
\text { SBC or project phase }\end{array}$ & $\begin{array}{l}\text { The costing study will capture costs in the RISE II intervention zones and will include } \\
\text { costs from the formative SBC program development stage and the first two years of } \\
\text { implementation. }\end{array}$ \\
\hline
\end{tabular}

as well as the capacity-building costs from Breakthrough ACTION and corresponding increased service costs delivered by a different implementing partner, also funded by USAID, are important to include for future planning, as funding sources may vary as programs mature. Costs associated with volunteer time, along with donated goods and services, are also important to include.

\section{Principle 3: Defining the cost types}

This principle distinguishes financial cost-the amount a funder or implementing organization pays for an SBC intervention-from economic cost, which includes opportunity costs of individual clients. As with defining the costing study perspective, reviewing the inclusion of opportunity costs is important when initiating a costing study. RISE II costing will assess economic costs, which includes the calculation of financial costs as a subset, to fulfill the dual purposes of the costing study-to inform budgeting and planning and to assess cost-effectiveness.

Differences between the financial and economic costs of RISE II will occur when some inputs are donated or are obtained at reduced price. Opportunity costs included in RISE II's full economic costs will include:

- Donated, unpaid, or under-paid labor attributed to community health volunteers or other program volunteers,

- Donated goods and services, which can include free or reduced mass media costs, transportation costs, and supplies, and

- Donated space for community meetings or other activities.

Because the planned CEA will not employ a comprehensive societal perspective, client opportunity costs 
associated with their intervention participation time will not be included-because including these types of opportunity costs would over-estimate how much program funding is required. When presenting the CEA results, both the financial costs (i.e. amount spent) and economic costs (including costs associated with donated labor, goods, and space) will be detailed, which will help other donors and implementers estimate the costs of similar interventions in other contexts. Other SBC costing studies have presented both financial and economic costs. ${ }^{12-14}$

\section{Principle 4: Defining unit costs}

This principle instructs analysts to clearly define what unit of measurement, or denominator, will be used in their unit cost calculations. While different kinds of denominators for SBC are reported in the literature, two of the more common units are:

- Costs per person exposed to mass media, community awareness raising activities (e.g., loudspeaker announcements), and other one-way forms of communication; and

- Costs per person participating in two-way communication, such as individual or group IPC.

These denominators will be most relevant for the RISE II costing activity. For one-way communication like mass media, determining the number of persons exposed will be based on endline survey data, when respondents are asked whether they heard SBC messages promoted during intervention activities. For two-way communication, the number participating will be assessed from program attendance records. For the CEA, the most important unit is the cost per health outcome, as discussed in Principle 9.

\section{Principle 5: Determining the appropriate time frames}

For any CEA, the time frame over which the evaluation is carried out should be long enough to capture all relevant costs and program effects. The first year of RISE II was focused on designing the intervention activities, with implementation beginning in the second year. Although two years of RISE II intervention activities are planned between its baseline and endline surveys, its SBC programming and resultant program impacts are expected to continue for approximately two years following the endline survey. As such, it will be important to allocate the design phase costs occurring prior to implementation over the entire project period. Actual cost and costeffectiveness analyses will only be available for the first three years, but a longer-term, projected CEA is potentially feasible, based on projected costs utilizing unit cost estimates and projected impacts that are based on trends from the baseline and endline surveys. 


\section{SECTION 2 \\ Resource Use Measurement}

The second section of the costing guidelines directs researchers to examine more specific questions about what cost components will be included, how costs will be allocated, and how they will be obtained and measured.

\section{6-Determining the scope of costs}

7-Measuring and allocating resources

\section{8-Sampling}

9-Measuring "units" of outputs

10-Timing data collection

\section{Principle 6: Determining the scope of the costs}

This principle suggests that analysts define the scope of the inputs included in the cost estimation and justify their inclusion in relation to the costing exercise's purpose, perspective, type of costs, and timeframe of the costing. Essentially, this principle requires definition of what is "in" or "out" of the scope of the costing. Following UCSR's structure, included SBC cost components can be further classified as personnel, recurrent, and capital costs.

\section{Personnel}

- RFSA direct personnel salaries

- RFSA support personnel salaries (e.g., accounting, finance, human resources)

- Breakthrough ACTION personnel salaries

- Other personnel costs (e.g., government salaries for participating in meetings)

- Donated volunteer time

- Consultants

\section{Recurrent}

- Office supplies (e.g., stationary, printer toner)

- Petty cash

- Rent and utilities (e.g., electric, gas, water)

- Cleaning

- Security

- Printing/production of SBC materials

- Mass media airtime (paid)

- Mass media airtime (donated, based on expected costs)

- Equipment less than $\$ 100$ and/or less than one year of utility

- Training costs-for recurrent training

- Meeting/workshop costs-related to implementation

- Per diems

- Transportation-associated with program implementation training and meetings

\section{Capital}

- Office space (included any donated space)

- Vehicles

- Equipment more than $\$ 100$ and that can be utilized more than one year

- Meeting and training costs associated with program design/start-up

The SBC activity unit costs will be limited to the design and implementation costs listed above. For the CEA calculations, however, an increase in the health behaviors targeted under RISE II (e.g., FP, ANC, facility-based deliveries) will generate additional service delivery costs for CEA calculations, such as increases in clinic visits, that may be funded by USAID under a separate project. Consequently, it is relevant to consider incorporating the estimated cost of those services in the cost-effectiveness calculation, and CEA estimates will be calculated with and 
without service delivery costs, for comparison purposes. Costs associated with improvements in key indicators will be calculated in the Lives Saved Tool (LiST) costing module within Spectrum and adjusted as needed after interviews with RFSAs and service delivery partners.

Because this application is considering less than a full societal perspective, the costing study excludes certain costs:

- Opportunity cost of client time for participation in SBC programs and receiving services

- Client out-of-pocket fees

- Donor management costs

- Any evaluation, learning, or knowledge management costs incurred by Breakthrough ACTION or Breakthrough RESEARCH (excepting direct contributions to the functioning of interventions), including:

- Evaluation efforts that track project progress beyond routine monitoring

- International and national dissemination activities (conferences, brochures, briefs, etc.), and

- Advocacy activities unrelated to the functioning of the interventions

Breakthrough RESEARCH's SBC costing guidelines also emphasize the importance of "above site" costs, those costs associated with contributions that are incurred above the site level, such as the central management costs of the RFSAs.

\section{Principle 7: Measuring and allocating resources}

This principle emphasizes the importance of clearly describing the methods used to estimate quantities of inputs and data sources. As with many costing exercises, the RISE II CEA will require mixed methods study design. Survey methods can measure use of time by volunteers and workers from other organizations. Costing activities will include:

- Review of Breakthrough ACTION and RFSA historical expenditure data. To assign a cost to those direct and indirect resources attributable to SBC interventions, such as staff time, travel, resources, workshops, and other recurrent costs, we will draw on expenditure data from Breakthrough ACTION's and the RFSAs' budget and cost accounting systems.

- Structured interviews with key individuals involved in the SBC program design and implementation.

Interviewers will follow a structured interview guide to solicit information about the design process, timeline, and the associated types and amounts of resources (staff and consultant time, travel, materials, workshops, research, etc.). Interviewers will administer this questionnaire to Breakthrough ACTION and RFSA central staff in Niger involved in program design and implementation.

- Structured interviews to assign costs to contributions by organizations other than Breakthrough ACTION and RFSAs. Resources other than those from Breakthrough ACTION that contributed to the design process will also be costed, such as time spent by government counterparts, or in-kind and other contributions from other partners. This information will be documented in the structured interviews, and supplemented by data on prices such as partner salaries, where feasible.

Principle 7 also encourages analysts to be explicit about methods of allocating joint costs. In RISE II, costs will be allocated to different SBC activities. If individuals work on multiple activities, their personnel time will be allocated according to percent estimates of their total time, per each activity. Allocating costs of design workshops to multiple activities will require assumptions about the proportions of effort for each activity.

\section{Principle 8: Sampling}

This principle discusses the use of sampling for estimating costs associated with service delivery. In theory, this could include observations of a sample of group IPC meetings to assess costs by documenting personnel, time, space, and materials required for each meeting. The RISE II CEA does not plan on sampling, nor interacting with clients, because costs will be obtained from implementing organizations, and client costs (i.e., value of their time participating in SBC interventions) are not being included as a cost component. As such, this principle is not applicable for the RISE II costing study. 


\section{Principle 9: Measuring "units" of output}

Principle 9 counsels the researcher to describe the selection of the data source(s) and methods for estimating the 'units' for unit costs and report potential biases. There are two purposes of the RISE II study: (1) examine cost-effectiveness associated with SBC health programming in RISE II; and (2) estimate unit costs for included SBC interventions for inclusion in the UCSR. For unit costs, the denominators will be per person exposed to one-way forms of communication (e.g., radio, community announcements) and per person participating in activities allowing for two-way communication (e.g., individual and group (PC). Data on exposure and participation will come from program records, monitoring and evaluation reports, and the endline survey.

A common health metric for multiple health behaviors is necessary for the CEA. In the RISE II program, there are seven primary health behaviors of interest:

- Modern contraceptive use

- ANC utilization
- Facility-based deliveries

- Breastfeeding

- Nutrition

- Handwashing

- Delay of marriage

Improvements in these health behaviors can be translated to improvements in health outcomes. When comparing multiple health areas, such as with integrated SBC, one can use disability-adjusted life years (DALYS) as a common metric: A DALY is one year of life lost due to ill health, disability, or early death. Breakthrough RESEARCH'S RISE II baseline and endline survey data will allow exploration of changes in behavioral health outcomes due to SBC interventions. These changes in behaviors will then be entered into the Lives Saved Tool (LIST) model in Spectrum to calculate neonatal, maternal, and child lives saved, which will then be converted to DALYs. Additional modeling will capture DALYs related to improvements in delayed marriage. Table $\mathbf{3}$ describes how each behavior will be captured for modeling.

\section{TABLE 3 RISE II HEALTH BEHAVIOR INDICATORS}

\section{INDICATOR}

\section{BASELINE}

Modern contraceptive use $x$

Percentage of women using modern contraception

\section{ANC use}

Percentage pregnant women attending at least 1 ANC visit

Percentage pregnant women attending at least 4 ANC visits

\section{Facility-based deliveries}

Percent of pregnancies with a facility-based birth

\section{Breastfeeding}

Percent of births with early introduction of breastfeeding

Percent of mothers breastfeeding-exclusive 0-6 months

Nutrition
$\begin{aligned} & \text { Percent of pregnant women with adequate nutrition } \\ & \text { Percent of children with adequate nutrition }\end{aligned}$
$\begin{aligned} & \text { Handwashing } \\ & \text { Percent of women reporting handwashing with soap }\end{aligned}$
$\begin{aligned} & \text { Delay of marriage } \\ & \text { Percent of females under age } 20 \text { married }\end{aligned}$

X $\quad$ X Spectrum: LiST

$X$

X

Spectrum: LiST

Spectrum: LiST

Spectrum: LiST

$x \quad x$

X Spectrum: FamPlan

$x$




\section{Principle 10: Timing of data collection}

This principle advises analysts to schedule data collection to minimize recall bias and, where relevant, account for seasonality and other differences over time. With SBC design and planning having occurred most recently, SBC intervention design costs of RISE II will be documented first, to forestall any recall bias.

Costing for data collection should weigh the benefits of frequent data collection and the administrative burden to organizations involved in the costing. The RISE II costing study proposes immediate data collection of SBC design costs, preferably before the baseline survey, to take full advantage of recent memory and existing documentation of meetings, workshops, conference calls, and personnel time spent developing and planning the SBC interventions. After implementation begins, data collection is proposed at six to eight months afterwards, for initial analysis. A second data collection is proposed at months 18 to 20 , with final data collection planned in conjunction with the endline survey (month 24). 


\section{SECTION 3 \\ Pricing and valuation}

Whereas Section 2 focuses on identifying and measuring SBC intervention cost components, this section focuses on specific elements of putting monetary values on these components.

11-Determining the source of price data

12-Valuing capital inputs

13-Selecting discount, inflation, and currency conversion rates

14-Using shadow prices

\section{Principle 11: Determining the source of price data}

Principle 11 advises analysts to choose data sources that reflect prices relevant to the purpose of the SBC costing and to describe prices and costs in a way that allows for adjustment across settings. In this application, most price data are expected to come from expenditure data, but other data sources for pricing may be used, including government and organizational pay scales, invoices, purchasing and shipping orders, and local market price lists.

Because costing informs CEAs, prices that reflect all financial and some donated costs are used as key inputs to calculate results for both financial and economic incremental cost effectiveness ratios (ICERs). While volunteer labor may be used in RISE II to provide some SBC interventions, the value of that labor will be needed if a comparable intervention is planned at a different time or location, where volunteer labor is not possible. Opportunity costs of volunteer time and in-kind donations such as donated airtime are estimated using "shadow" prices, as described in Principle 14.

\section{Principle 12: Valuing capital inputs}

This principle advises on how a costing study should appropriately depreciate capital inputs (those inputs with a useful life of greater than one year) to reflect their cost over the relevant timeframe. Based on overall organizational budgets, for the RISE II program a subset of office space, vehicles, building improvements, and other shared capital expenses will be apportioned, along with other key capital costs including SBC design costs, some training, and mass media messaging. Useful lifespans for these resources will be assigned to amortize those costs using the appropriate local discount rate.

\section{Principle 13: Selecting discount, inflation, and currency conversion rates}

According to this principle, cost analysts must use appropriate methods for considering inflation and exchange rates. The RISE II study will value inputs in local currency or US dollars as appropriate and show results in both local currency and US dollars for comparison, using average exchange rates for relevant periods. To adjust for inflation, all costs will be reported in constant prices, using an appropriate month and year (e.g., January 2020) as a base. Recognizing the global interest in these results, they will be reported in both US dollars and international dollars, using purchasing power parity international dollar exchange rates according to World Bank methodology.

\section{Principle 14: Using shadow prices}

Principle 14 encourages analysts to report how they assign a "shadow" price to contributions that do not have a market price, such as volunteer time. Community volunteer time will be valued using the local minimum wage or prevailing local cost of unskilled labor. Donated time by any professional will be valued at market rates, and donated airtime will be valued at the rate at which RISE II pays for broadcast announcements and programs. 


\section{SECTION 4}

\section{Analyzing and Presenting Results}

The final section addresses analysis and presentation of costing results. In the SBC costing literature, numerous instances of unclear documentation of interventions, as well as their steps of analyses, make useful interpretation difficult, in addition to hindering collation of results for summary analysis. Adherence to the SBC costing guidelines in this regard should lead to improvements in the literature and our overall understanding of SBC costs.

15-Exploring cost functions and heterogeneity

16-Dealing with uncertainty

17-Employing transparency

\section{Principle 15: Exploring cost functions and heterogeneity}

Principle 15 addresses intervention cost variations by site size, organization, sub-populations, or other heterogeneity factors. Due to RISE II's complex operational environment, its integrated SBC campaign costs are not readily transferrable or easily translatable to other locations, even when adjusting for local market prices. Scale is another important consideration, as the unit costs for delivering SBC interventions are not likely the same when reaching 1,000 people versus 100,000. Although these are important factors, the RISE II costing study will not explore cost functions.

\section{Principle 16: Dealing with uncertainty}

This principle emphasizes how the results of any cost study depend, to a significant extent, on the assumptions that are used to calculate costs and outputs. A sensitivity analyses will assess the extent to which changes in assumptions substantially alter results for RISE II. An initial sensitivity analysis will allow for uncertainty of both costs and efficacy of RISE II's SBC interventions. Some of the costing assumptions where uncertainty may exist and thus sensitivity analysis will be necessary include:

1. the proportion of time allocated to SBC activity design,

2. allocation of capital and recurrent expenses to SBC activities,

3. the number of contributed volunteer hours, and

4. "shadow" prices associated with donated supplies and labor.

As additional assumptions or areas of uncertainty are identified, they will be flagged for sensitivity analysis. For sensitivity analysis regarding effectiveness, the $95 \%$ confidence interval for the percent increase in health behaviors can be used for sensitivity testing. RISE II costing results will present both cost-effectiveness findings along with sensitivity results, from the least favorable (lowest impact, highest cost) to most favorable (highest impact, lowest cost) outcomes.

\section{Principle 17: Employing transparency}

The final principle states that cost analyses should clearly articulate both a study's methods and assumptions, as minimum reporting standards. Clear, unambiguous reporting of cost findings and underlying assumptions is especially critical, due to varied and different potential audiences, and potential for misinterpretation of results.

RISE II's study methods and findings will be reported to key stakeholders in Niger (e.g., RFSAs, Breakthrough ACTION, USAID, Ministry of Health) as well as more broadly, for planning purposes, and these results will contribute to Breakthrough RESEARCH's SBC cost repository workbook and UCSR online so other cost researchers can examine the inputs, methods, and results for their own benchmarking and planning. ${ }^{11,15}$ The rigor of the RISE II CEA study design is expected to provoke substantial interest in its findings among the global community, with potential for publishing in a peer-reviewed journal. 


\section{Conclusions}

Applying Breakthrough RESEARCH's SBC costing guidelines to the RISE II program in Niger allowed for a systematic review of the important considerations for how a costing and cost-effectiveness study should be conducted in this context. The results will inform the forthcoming study protocol, where the findings will contribute to the plan for how the CEA will be conducted. Most of the SBC guidelines are relevant to the RISE II program, and those not relevant are due to the specifics of the CEA, which will neither sample nor conduct program heterogeneity analysis. Of all the principles articulated in the guidelines, the most important for the RISE II CEA is defining the SBC interventions for health and their associated cost components, allocating costs across activities and time, and measuring health outputs from improved health behaviors.

Additionally, this document can serve as an example on how to systematically apply the costing guidelines to other SBC case studies in preparation for costing studies. Future case study applications can help build the evidence base on SBC costs and cost-effectiveness. Further applications can also provide insights on how the costing guidelines can be expanded and improved to account for specific circumstances that are not currently represented. 


\section{References}

1. Rosen, J.E., W. DeCormier Plosky, and L. Bollinger. 2019. "Guidelines for costing of social and behavior change health interventions," Breakthrough RESEARCH. Washington DC: Population Council. Available at: https://breakthroughactionandresearch.org/wp-content/uploads/2019/10/ guidelines-for-costing-sbc-interventions.pdf

2. USAID. 2018. "Social behavior change." Available at: https:// www.usaid.gov/what-we-do/global-health/cross-cutting-areas/social-and-behavior-change. Accessed August 28, 2020.

3. CDC. 2019. "Cost-effectiveness analysis (CEA)." Available at: https://www.cdc.gov/policy/polaris/economics/cost-effectiveness.html. Accessed August 28, 2020.

4. Health Communication Capacity Collaborative. 2017. "Integrated SBCC programs implementation kit." Available at: https://www.thecompassforsbc.org/sbcc-tools/integrated-sbcc-programs-implementation-kit. Accessed August 28, 2020.

5. USAID. 2018. "Resilience in the Sahel Enhanced (RISE) II technical approach working paper." Available at: https:// www.usaid.gov/documents/1860/usaid-resilience-sahel-enhanced-rise-ii-technical-approach-working-paper. Accessed August 28, 2020.

6. Breakthrough RESEARCH. 2020. "Study protocol: Breakthrough Research evaluation of RISE II integrated social and behavior change activities in Niger."

7. Vassall, A. et al. 2017. "Reference case for estimating the costs of global health services and interventions." Available at: https://ghcosting.org/pages/standards/reference_case. Accessed August 28, 2020.

8. Homan R. 2016. "Costing of social norm interventions: A primer for the Passages project." Available at: http://irh.org/ wp-content/uploads/2016/10/Costing_Social_Norm_Interventions_Passages.pdf. Accessed August 28, 2020.

9. Breakthrough RESEARCH. 2019. "The added value of costing social and behavior change interventions," Research to Practice Brief. Washington, DC: Population Council. Available at: https://breakthroughactionandresearch.org/wp-content/uploads/2019/10/Costing-Brief.pdf

10. Unit Cost Study Repository. 2020. Available at: https://ghcosting.org/pages/data/ucsr/app/. Accessed August 28, 2020

11. World Health Organization. 2016. "Key health indicators: Niger." Available at: https://apps.who.int/gho/data/node. cco.ki-NER?lang=en. Accessed August 28, 2020.

12. Fung, I. C. H. et al. 2007. "Modelling the impact and cost-effectiveness of the HIV intervention programme amongst commercial sex workers in Ahmedabad, Gujarat, India," BMC Public Health 7: 195. doi: 10.1186/1471-2458-7-195

13. Manzi, F. et al. 2008. "From strategy development to routine implementation: The cost of intermittent preventive treatment in infants for malaria control," BMC Health Services Research 8: 165. doi: 10.1186/1472-6963-8-165
14. Terris-Prestholt, F. et al. 2006. "From trial intervention to scale-up: Costs of an adolescent sexual health program in Mwanza, Tanzania," Sexually Transmitted Diseases 33(1): S133-S139. doi: 10.1097/01.olq.0000200606.98181.42

15. Breakthrough RESEARCH. 2020. SBC Cost Data Repository. Available upon request at nbellows@avenirhealth.org. 


\section{Population Council}

4301 Connecticut Ave., NW | Suite 280

Washington, DC 20008

+12022379400

breakthroughactionandresearch.org 